\title{
FUNCTIONAL DIFFERENTIAL EQUATIONS DETERMINING STEADY SIZE DISTRIBUTIONS FOR POPULATIONS OF CEIIS GROWING EXPONENTIAILY
}

\author{
A. J. HALL ${ }^{1}$ AND G. C. WAKE ${ }^{2}$
}

(Received 10 May 1989)

\begin{abstract}
A population of cells growing and dividing often goes through a phase of exponential growth of numbers, during which the size distribution remains steady. In this paper we study the function differential equation governing this steady size distribution in the particular case where the individual cells themselves are growing exponentially in size. A series solution is obtained for the case where the probability of cell division is proportional to any positive power of the cell size, and a method for finding closed-form solutions for a more general class of cell division functions is developed.
\end{abstract}

\section{Introduction}

If we consider a population of cells which are all growing and dividing, we may define a cell number density $n(x, t)$ such that $n(x, t) d x$ gives the number of cells with a size between $x$ and $x+d x$ at time $t$. Biologists are interested in the steady size distributions (SSDs) which can arise where the total number of cells in the population increases exponentially, but the proportion of cells in any size class remains the same (Collins and Richmond [2]; Tyson and Diekmann [8]). In our introduction to an earlier paper (Hall and Wake [3]), we discussed the situation in which all cells of size $x$ have the same time-invariant growth rate $g(x)$ and division rate $b(x)$ (such that $b(x) d t$ is the proportion of cells of size $x$ which will divide in time $d t$ ), and where on division each mother cell divides into $\alpha$ daughter cells each of the same size.

\footnotetext{
${ }^{1}$ Plant Physiology Division, DSIR, Palmerston North, New Zealand and Department of Mathematics and Statistics, Massey University, Palmerston North, New Zealand.

${ }^{2}$ Department of Mathematics and Statistics, Massey University, Palmerston North, New Zealand.

(C) Copyright Australian Mathematical Society 1990, Serial-fee code 0334-2700/90
} 
We showed that if we write $n(x, t)=y(x) N(t)$, where $N(t)$ is the total population at time $t$ and $y(x)$ is the probability density function describing the SSD, then $N(t)$ is indeed an exponential function of time, and the functional differential equation for the $\operatorname{SSD} y(x)$ is

$$
\frac{d}{d x}(g(x) y(x))=-[b(x)+Q] y(x)+\alpha^{2} b(\alpha x) y(\alpha x)
$$

where $Q$ is a functional on $y(x)$ defined by

$$
Q=(\alpha-1) \int_{0}^{\infty} b(x) y(x) d x .
$$

Equation (1) is also given in Tyson and Diekmann [8] for the biologically realistic case of $\alpha=2$, which represents reproduction by binary fission with two daughter cells of equal size. The size of a cell can be taken to mean any property which is distributed evenly between the daughter cells when a mother cell divides. For example, $x$ could refer to the volume, mass, or perhaps even DNA content of the cells, or in cases where cell extension and division occur in just one dimension it may be appropriate to treat $x$ as the cell length.

All cells must be of positive size, so we may set $y(x) \equiv 0$ for $x<0$, and as $y(x)$ is a probability density function it must satisfy the conditions

$$
\int_{0}^{\infty} y(x) d x=1
$$

and

$$
y(x) \geq 0 \text {. }
$$

In this paper we set out to solve equations (1) and (2) for $y(x)$ subject to conditions (3) and (4), assuming that the functions $g(x)$ and $b(x)$ are given. The solution to an "inverse" problem of finding $g(x)$ from an observed SSD $y(x)$ and the size distribution of dividing cells $\left(y_{m}(x)\right.$ say) has been given by Collins and Richmond [2], and Powell [7] showed how $y(x)$ can be deduced from $y_{m}(x)$ if $g(x)$ is given in the form $g(x)=g x$. Tyson and Diekmann [8] present the solution to our "forward" problem for general $g(x)$, but with $b(x)$ restricted to forms such that there exists a maximum size $\left(x_{2}\right)$ and minimum size $\left(x_{1}\right)$ for cell division with $x_{2} \leq 2 x_{1}$. Here we restrict our attention to the case $g(x)=g x$ considered by Powell [7] and Koch and Schaechter [6], and hence solve equation (1) to (4) for forms of $b(x)$ more general than those considered by Tyson and Diekmann. The quantity $Q$ can be given a number of interpretations. Hall and Wake ([3]) showed that $Q$ as defined by (2) also satisfies

$$
d N / d t=Q N(t)
$$


where $N(t)$ is the total population at time $t$, so $Q$ can be regarded as the exponential rate of growth of the population. Also, the rate at which the total size of all the individuals in the population increases can be found either by integrating first over all sizes to find the total size then differentiating with respect to $t$, or simply by integrating the growth rate over all individuals in the population. This gives

$$
\frac{d}{d t}\left[\int_{0}^{\infty} x N(t) y(x) d x\right]=\int_{0}^{\infty} N(t) y(x) g(x) d x,
$$

or

$$
\frac{d N}{d t}\left[\int_{0}^{\infty} x y(x) d x\right]=N(t) \int_{0}^{\infty} y(x) g(x) d x,
$$

then using (5) we obtain

$$
Q=\frac{\int_{0}^{\infty} g(x) y(x) d x}{\int_{0}^{\infty} x y(x) d x}
$$

so $Q$ may also be regarded as ratio of the average growth rate of the individuals in the population to their average size.

1.1 The special case $g(x)=g x$

It is difficult to see how to obtain closed-form solutions to (1) subject to (3) and (4) in the most general case, most obviously because of the presence of the unknown functional $Q$. However, in two simple cases we can find $Q$ readily. The first of these is if $b(x)=b$, a constant, so that from (2) $Q=(\alpha-1) b$. A solution for this case with $g(x)$ also constant is given in Hall and Wake [3]. A solution with $b(x)$ constant can also be obtained for $g(x)=g x^{1-k}(k>0)$ by using the transformation $Z(x)=g(x) y(x)$ to obtain an equation of the same form as equation (21) below. However, a constant probability of division over all sizes is not plausible biologically, so this case is not discussed further in this paper.

The case where the cells themselves grow in size exponentially during their life cycle, so $g(x)=g x$ ( $g$ constant), is more plausible biologically, and has been discussed in the bacteriological literature. In this case, (7) simplifies immediately to

$$
Q=g
$$

Equation (1) then becomes

$$
g x y^{\prime}(x)=-[b(x)+2 g] y(x)+\alpha^{2} b(\alpha x) y(\alpha x),
$$

as given by Powell [7] and Koch and Schaechter [6].

In this paper we seek solutions to this equation, subject to the normalising and non-negativity conditions (3) and (4). 
It is trivial to show that

$$
y=\delta(x),
$$

where $\delta(x)$ is the Dirac delta function, is a generalised solution of (9) for all forms of $b(x)$, and in fact is a solution of (1) for any $g(x)$ such that $g(0)=0$. In fact, $y(x)=\delta(x)$ is the only non-negative generalised solution satisfying (3), and therefore the only generalised solution which may be regarded as a probability distribution. The existence of this solution is reasonable in that it says that if we have a population all of size zero at any time, this situation will not change provided $g(0)=0$.

We now look for classical solutions to (9) subject to conditions (3) and (4). Multiplying by $x$ and rearranging gives

$$
g\left[x^{2} y^{\prime}(x)+2 x y(x)\right]=-x b(x) y(x)+\alpha^{2} x b(\alpha x) y(\alpha x) .
$$

This suggests the change of variable

$$
Z(x)=x^{2} y(x)
$$

to give

Setting

$$
g Z^{\prime}(x)=-\frac{b(x)}{x} Z(x)+\frac{b(\alpha x)}{x} Z(\alpha x)
$$

$$
a(x)=\frac{b(x)}{g x}
$$

simplifies (13) to

$$
Z^{\prime}(x)=-a(x) Z(x)+\alpha a(\alpha x) Z(\alpha x) .
$$

This equation needs to be solved subject to $Z(x) \geq 0$ and either of the conditions

$$
\int_{0}^{\infty} \frac{Z(x)}{x^{2}} d x=1
$$

or

$$
\int_{0}^{\infty} a(x) \frac{Z(x)}{x} d x=\frac{1}{\alpha-1} \text {. }
$$

Here the second condition follows either from $Q=g$, or from integration by parts and substituting from (15). Equations (15) and (16) together are equivalent to (15) and (17) together.

Note that equation (15) can be written in the integral form

$$
Z(x)=\int_{x}^{\alpha x} a(s) Z(s) d x,
$$

which despite superficial similarities is very different from the classical Volterra integral equation. It follows that

$$
Z(0)=0 \text {, }
$$


then substitution into (15) and its derivatives shows that

$$
Z^{(n)}(0)=0, \forall n \geq 0 \text {. }
$$

Hence any solution to (15) must be non-analytic ("infinitely flat") at the origin.

In the next three sections we examine solutions of (15) subject to (16) or (17) for different forms of $b(x)$. We find solutions involving series of exponentials for the two cases $b(x)=b x^{k}$ and $b(x)=b x^{k} H\left(x-x_{1}\right)$, and then describe a general method of solution in cases where $b(x)$ is zero below some minimum size for division $x_{1}$, and becomes infinite at some maximum cell size $x_{2}$.

\section{The case $b(x)=b x^{k}$}

In this case, we have $a(x)=b x^{k} / g x=a x^{k-1}$ say (where $a=b / g$ ), so that (15) becomes

$$
Z^{\prime}(x)=x^{k-1}\left(-a Z(x)+a \alpha^{k} Z(\alpha x)\right) .
$$

If we make the substitutions $z=x^{k} / k$ and $Y(z)=Z(x)$, so $Z^{\prime}(x)=$ $Y^{\prime}(z) d z / d x=x^{k-1} Y^{\prime}(z)$ and $Z(\alpha x)=Y\left((\alpha x)^{k} / k\right)=Y\left(\alpha^{k} z\right)$, then (21) becomes (after cancellation of $x^{k-1}$ )

$$
Y^{\prime}(z)=-a Y(z)+a \alpha^{k} Y\left(\alpha^{k} z\right)
$$

This equation is in the same form as that dealt with in Hall and Wake [3] and Kato and McLeod [5], so we can write down the solution immediately. In terms of our variables this is

$$
Y(z)=C \sum_{n=0}^{\infty} \frac{(-1)^{n} \alpha^{k n}}{\left(\alpha^{k}-1\right) \cdots\left(\alpha^{k n}-1\right)} e^{-a \alpha^{k n} z},
$$

where by convention the expression in the denominator is given the value 1 when $n=0$. This solution is unique apart form the multiplicative constant $C$. Now reverting to our $Z$ and $x$ notation we obtain

$$
Z(x)=C \sum_{n=0}^{\infty} \frac{(-1)^{n} \alpha^{k n}}{\left(\alpha^{k}-1\right) \cdots\left(\alpha^{k n}-1\right)} e^{-a \alpha^{n k} x^{k} / k}
$$

The probability density function for the SSD is then given (from 12)) by

$$
y(x)=\frac{Z(x)}{x^{2}}
$$

with the constant $C$ to be evaluated from condition (17), which can be written in this case as

$$
\int_{0}^{\infty} x^{k-2} Z(x) d x=\frac{1}{a(\alpha-1)}
$$


Note that for $k=0$ the transformation, and therefore the solution, is illdefined, and for $k<0$ the series does not converge. The solution is therefore valid only for

$$
k>0 \text {. }
$$

Obtaining the solution (24) was straightforward because we managed to transform the original problem into one for which the solution was known. However, using the integral condition (26) to find the constant $C$ is not so easy, as it involves carrying out the integration in

$$
\int_{0}^{\infty} x^{k-2} Z(x) d x=C \int_{0}^{\infty}\left[\sum_{n=0}^{\infty} \frac{(-1)^{n} \alpha^{k n}}{\left(\alpha^{k}-1\right) \cdots\left(\alpha^{k n}-1\right)} x^{k-2} e^{-a^{\alpha^{n k} x^{k} / k}}\right] d x
$$

for all $k>0$.

For $0<k \leq 1$, care must be taken over interchanging the integral and summation signs in this expression, as $\int_{0}^{\infty} x^{p} e^{-x} d x$ does not exist for $p \leq$ -1 . It is necessary to treat two cases separately: $k=m^{-1}$ for some integer $m$, and $k \neq m^{-1}$ for any such $m$.

2.1 The case $k \neq m^{-1}$

Firstly, consider the integral $\int_{0}^{\infty} x^{p} Z(x) d x$, where $p \neq-1$. Integrating by parts, we get

$$
\int_{0}^{\infty} x^{p} Z(x) d x=\left[\frac{x^{p+1}}{p+1} Z(x)\right]_{0}^{\infty}-\frac{1}{p+1} \int_{0}^{\infty} x^{p+1} Z^{\prime}(x) d x .
$$

Now the square-bracket term is zero because $Z$ is infinitely flat at the origin, and l'Hopital's rule can be used to show that $\lim _{x \rightarrow \infty} x^{p} Z(x)=0$ for all real $p$. We can then substitute from (21) for $Z^{\prime}(x)$ and use a simple change of variable to give

$$
\begin{aligned}
\int_{0}^{\infty} & x^{p} Z(x) d x \\
& =-\frac{1}{p+1}\left[-a \int_{0}^{\infty} x^{p+k} Z(x) d x+a \alpha^{k} \int_{0}^{\infty} x^{p+k} Z(\alpha x) d x\right],
\end{aligned}
$$

so

$$
\int_{0}^{\infty} x^{p} Z(x) d x=\frac{a\left(1-\alpha^{-(p+1)}\right)}{p+1} \int_{0}^{\infty} x^{p+k} Z(x) d x .
$$

If we apply this $m$ times, starting with $p=k-2$, (and remembering that $k m \neq 1, \forall m \in I^{+}$) we get

$$
\begin{aligned}
\int_{0}^{\infty} & x^{k-2} Z(x) d x \\
\quad= & \frac{a^{m}\left(1-a^{1-k}\right)\left(1-a^{1-2 k}\right) \cdots\left(1-\alpha^{1-m k}\right)}{(k-1)(2 k-1) \cdots(m k-1)} \int_{0}^{\infty} x^{(m+1) k-2} Z(x) d x .
\end{aligned}
$$


Now for all $k>0$, it is possible to choose $m \in I^{+}$such that $k>\frac{1}{m+1}$; for $k>1$ we would choose $m=0$. With such a choice of $m$, we can now substitute for $Z(x)$ and interchange the summation and integral signs. (Note that for $k<\frac{1}{m+1}$ the interchange is impossible):

$$
\begin{aligned}
\int_{0}^{\infty} x^{k-2} Z(x) d x= & C \frac{a^{m}\left(1 \alpha^{1-k}\right)\left(1-\alpha^{1-2 k}\right) \cdots\left(1-\alpha^{1-m k}\right)}{(k-1)(2 k-1) \cdots(m k-1)} . \\
& \cdot \sum_{n=0}^{\infty} \frac{(-1)^{n} \alpha^{k n}}{\left(\alpha^{k}-1\right) \cdots\left(\alpha^{k n}-1\right)} \int_{0}^{\infty} x^{(m+1) k-2} e^{-a \alpha^{n k} x^{k} / k} d x
\end{aligned}
$$

Making the natural substitution $z=\frac{a}{k} \alpha^{n k} x^{k}$ we get

$$
x^{m k-1}=(k / a)^{m-1 / k} \alpha^{n(1-m k)} z^{m-1 / k}
$$

and

so

$$
x^{k-1} d x=\frac{\alpha^{-n k}}{a} d z
$$

$$
\begin{aligned}
\int_{0}^{\infty} x^{k-2} Z(x) d x= & C(k / a)^{m-1 / k} \frac{a^{m-1}\left(1-\alpha^{1-k}\right)\left(1-\alpha^{1-2 k}\right) \cdots\left(1-\alpha^{1-m k}\right)}{(k-1)(2 k-1) \cdots(m k-1)} \\
& \cdot \sum_{n=0}^{\infty} \frac{(-1)^{n} \alpha^{n(1-m k)}}{\left(\alpha^{k}-1\right) \cdots\left(\alpha^{k n}-1\right)} \int_{0}^{\infty} z^{m-1 / k} e^{-z} d z
\end{aligned}
$$

To simplify this we define

$$
K(\alpha, \beta)=\sum_{n=0}^{\infty} \frac{(-1)^{n} \beta^{n}}{(\alpha-1) \cdots\left(\alpha^{n}-1\right)},
$$

and by definition

$$
\Gamma(p)=\int_{0}^{\infty} x^{p-1} e^{-x} d x
$$

so (35) can be written as

$$
\begin{aligned}
& \int_{0}^{\infty} x^{k-2} Z(x) d x \\
&= C(k / a)^{m-1 / k} \frac{a^{m-1} m\left(1-\alpha^{1-k}\right)\left(1-\alpha^{1-2 k}\right) \cdots\left(1-\alpha^{1-m k}\right)}{(k-1)(2 k-1) \cdots(m k-1)} \\
& \times K\left(\alpha^{k}, \alpha^{1-m k}\right) \Gamma\left(m+1-\frac{1}{k}\right) \\
&= C(k / a)^{-1 / k} \frac{\left(1-\alpha^{1-k}\right)\left(1-\alpha^{1-2 k}\right) \cdots\left(1-\alpha^{1-m k}\right)}{a\left(1-\frac{1}{k}\right)\left(2-\frac{1}{k}\right) \cdots\left(m-\frac{1}{k}\right)} \\
& \times K\left(\alpha^{k}, \alpha^{1-m k}\right) \Gamma\left(m+1-\frac{1}{k}\right) .
\end{aligned}
$$


Now we have

$$
\Gamma(m+1-1 / k)=(m-1 / k) \cdots(2-1 / k)(1-1 / k) \Gamma(1-1 / k)
$$

and, using (97) repeatedly (see appendix),

$$
K\left(\alpha^{k}, \alpha\right)=\left(1-\alpha^{1-k}\right)\left(1-\alpha^{1-2 k}\right) \cdots\left(1-\alpha^{1-m k}\right) K\left(\alpha^{k}, \alpha^{1-m k}\right),
$$

so (38) can be simplified to

$$
\int_{0}^{\infty} x^{k-2} Z(x) d x=C\left(\frac{k}{a}\right)^{-1 / k} \frac{1}{a} K\left(\alpha^{k}, \alpha\right) \Gamma\left(1-\frac{1}{k}\right) .
$$

So from the integral condition (17) we get

$$
C=\left(\frac{k}{a}\right)^{1 / k} \frac{1}{(\alpha-1) K\left(\alpha^{k} \alpha\right) \Gamma\left(1-\frac{1}{k}\right)}
$$

Note that using (96) (see appendix) we could write $K\left(\alpha^{k}, \alpha\right)$ in a somewhat neater form as the infinite product

$$
K\left(\alpha^{k}, \alpha\right)=\prod_{n=1}^{\infty}\left(1-\alpha^{1-k n}\right)
$$

\subsection{The case $\mathrm{km}^{-1}$}

For $k=\frac{1}{m}, m \in I^{+}$, if we start with $p=k-2$ we can only apply the iterative relationship (30) used in the previous section $m-1$ times, giving

$$
\begin{aligned}
\int_{0}^{\infty} & x^{k-2} Z(x) d x \\
& =\frac{a^{m-1}\left(1-\alpha^{1-k}\right)\left(1-\alpha^{1-2 k}\right) \cdots\left(1-\alpha^{1-(m-1) k}\right)}{(k-1)(2 k-1) \cdots((m-1) k-1)} \int_{0}^{\infty} x^{-1} Z(x) d x .
\end{aligned}
$$

The integral on the right can be integrated by parts once more, giving

$$
\begin{aligned}
\int_{0}^{\infty} & x^{-1} Z(x) d x=-\int_{0}^{\infty}(\log x) Z^{\prime}(x) d x \\
& =a\left[\int_{0}^{\infty}(\log x) x^{k-1} Z(x) d x-\alpha^{k} \int_{0}^{\infty}(\log x) x^{k-1} Z(\alpha x) d x\right] \\
& =a\left[\int_{0}^{\infty}(\log x) x^{k-1} Z(x) d x-\int_{0}^{\infty}(\log s-\log \alpha) s^{k-1} Z(s) d s\right],
\end{aligned}
$$

so

$$
\int_{0}^{\infty} x^{-1} Z(x) d x=a \log \alpha \int_{0}^{\infty} x^{k-1} Z(x) d x
$$


where once again we have used (15) to replace $Z^{\prime}(x)$. Now substitute for $Z(x)$ from (24) and interchange the integral and summation signs (which is now permitted) to give

$$
\begin{aligned}
\int_{0}^{\infty} x^{-1} Z(x) d x & =C a \log \alpha \sum_{n=0}^{\infty} \frac{(-1)^{n} \alpha^{k n}}{\left(\alpha^{k}-1\right) \cdots\left(\alpha^{k n}-1\right)} \int_{0}^{\infty} x^{k-1} e^{-a \alpha^{k n} x^{k} / k} d x \\
& =C \log \alpha \sum_{n=0}^{\infty} \frac{(-1)^{n}}{\left(\alpha^{k}-1\right) \cdots\left(\alpha^{k n}-1\right)} \int_{0}^{\infty} s e^{-s} d s,
\end{aligned}
$$

so

$$
\int_{0}^{\infty} x^{-1} Z(x) d x=C K\left(\alpha^{k}, 1\right) \log \alpha .
$$

Thus (44) becomes

$$
\begin{aligned}
\int_{0}^{\infty} & x^{k-2} Z(x) d x \\
& =C K\left(\alpha^{k}, 1\right) \log \alpha \frac{a^{m-1}\left(1-\alpha^{1-k}\right)\left(1-\alpha^{1-2 k}\right) \cdots\left(1-\alpha^{1-(m-1) k}\right)}{(k-1)(2 k-1) \cdots((m-1) k-1)} .
\end{aligned}
$$

Applying the integral condition (26) leads to

$$
C=\frac{(k-1)(2 k-1) \cdots((m-1) k-1)}{\left(1-\alpha^{1-k}\right)\left(1-\alpha^{1-2 k}\right) \cdots\left(1-\alpha^{1-(m-1) k}\right)} \cdot \frac{1}{a^{m}(\alpha-1) K\left(\alpha^{k}, 1\right) \log \alpha} .
$$

This can be written a little more tidily by (taking $m$ factors of $k$ out of the numerator and using $m=1 / k$. Then

$$
C(k / a)^{1 / k} \frac{m !}{\left(\alpha^{k}-1\right)\left(\alpha^{2 k}-1\right) \cdots\left(\alpha^{m k}-1\right)} \cdot \frac{1}{K\left(\alpha^{k}, 1\right) \log \alpha},
$$

where of course $m k=1$.

\subsection{Discussion and examples}

We have shown that the SSD in the case $g(x)=g x$ and $b(x)=b x^{k}$ is

$$
y(x)=\frac{C}{x^{2}} \sum_{n=0}^{\infty} \frac{(-1)^{n} \alpha^{k n} e^{-a^{a^{n k} x^{k} / k}}}{\left(\alpha^{k}-1\right) \cdots\left(\alpha^{k n}-1\right)} .
$$

where $a=b / g$ and

$$
C= \begin{cases}(k / a)^{1 / k} \frac{m !}{\left(\alpha^{k}-1\right)\left(\alpha^{2 k}-1\right) \cdots\left(\alpha^{m k}-1\right)} \frac{1}{K\left(\alpha^{k}, 1\right) \log \alpha}, & k=\frac{1}{m}, m \in I^{+} \\ (k / a)^{1 / k} \frac{1}{(\alpha-1) K\left(\alpha^{k}, \alpha\right) \Gamma\left(1-\frac{1}{k}\right)}, & k \neq \frac{1}{m}, m \in I^{+} .\end{cases}
$$

The form of $C$ for $k=1 / m$ can be shown to be equal to the limit of the form for $k \neq 1 / m$ as $k \rightarrow 1 / m$ by expanding the gamma function, expressing 
$K\left(\alpha^{k}, \alpha\right)$ in terms of $K\left(\alpha^{k}, 1\right)$, then using the L'Hopital limit

$$
\lim _{m k \rightarrow 1}\left(1-\alpha^{1-m k}\right) /(m k-1)=\log \alpha \text {. }
$$

Hence $C$ is a continuous function of $k$.

When $k$ is an integer, we can obtain the $k$ th moment of the SSD about the origin, $\mu_{k}=\int_{0}^{\infty} x^{k} y(x) d x$, directly from the integral condition (26) as

$$
\mu_{k}=\frac{1}{a(\alpha-1)}
$$

An iterative expression for moments beyond the $k$ th is obtainable by applying a Mellin Transform to (21): multiplying through by $x^{m-1}$ and integrating over all $x$ ), remembering that $y(x)=x^{-2} Z(x)$ gives

$$
\int_{0}^{\infty} x^{m-1} Z^{\prime}(x) d x=-a \mu_{m+k}+a \int_{0}^{\infty} \alpha^{k} x^{m+k}\left[x^{-2} Z(\alpha x)\right] d x .
$$

Integration by parts on the left and the substitution $z=\alpha x$ on the right then leads to

$$
-(m-1) \mu_{m}=-a \mu_{m+k}+a \alpha^{-(m-1)} \mu_{m+k},
$$

so we have the iterative equation

$$
\mu_{m+k}=\frac{(m-1)}{a\left(1-\alpha^{-(m-1)}\right)} \mu_{m}
$$

provided $m>1$. This enables us to deduce all the moments of the SSD once we have found the $k$ th moment by using (52) and all other moments up to the $k+1$ th by integration of (50). For example, in the particular case $k=1$, the mean is

$$
\mu=\frac{1}{a(\alpha-1)}
$$

and the second moment about the origin $\left(\mu_{2} \int_{0}^{\infty} x^{2} y(x) d x=\int_{0}^{\infty} Z(x) d x\right)$ can be obtained by integrating (24) term by term to give

$$
\mu_{2}=\frac{1}{a^{2}(\alpha-1) \log \alpha} .
$$

We can then apply (55) repeatedly to obtain

$$
\mu_{n}=\frac{(n-2) !}{a^{n}(\alpha-1) \log \alpha \prod_{k=1}^{n-2}\left(1-\alpha^{-k}\right)}, \quad n>2 .
$$

Figure 1 shows the shape of the SSD function $y(x)$ with $a=1$ and $\alpha=2$ for a range of values of $k$. Consideration of the differential equation formed in the extreme cases shows that as $k \rightarrow 0$, the SSD $y(x)$ approaches the Dirac 


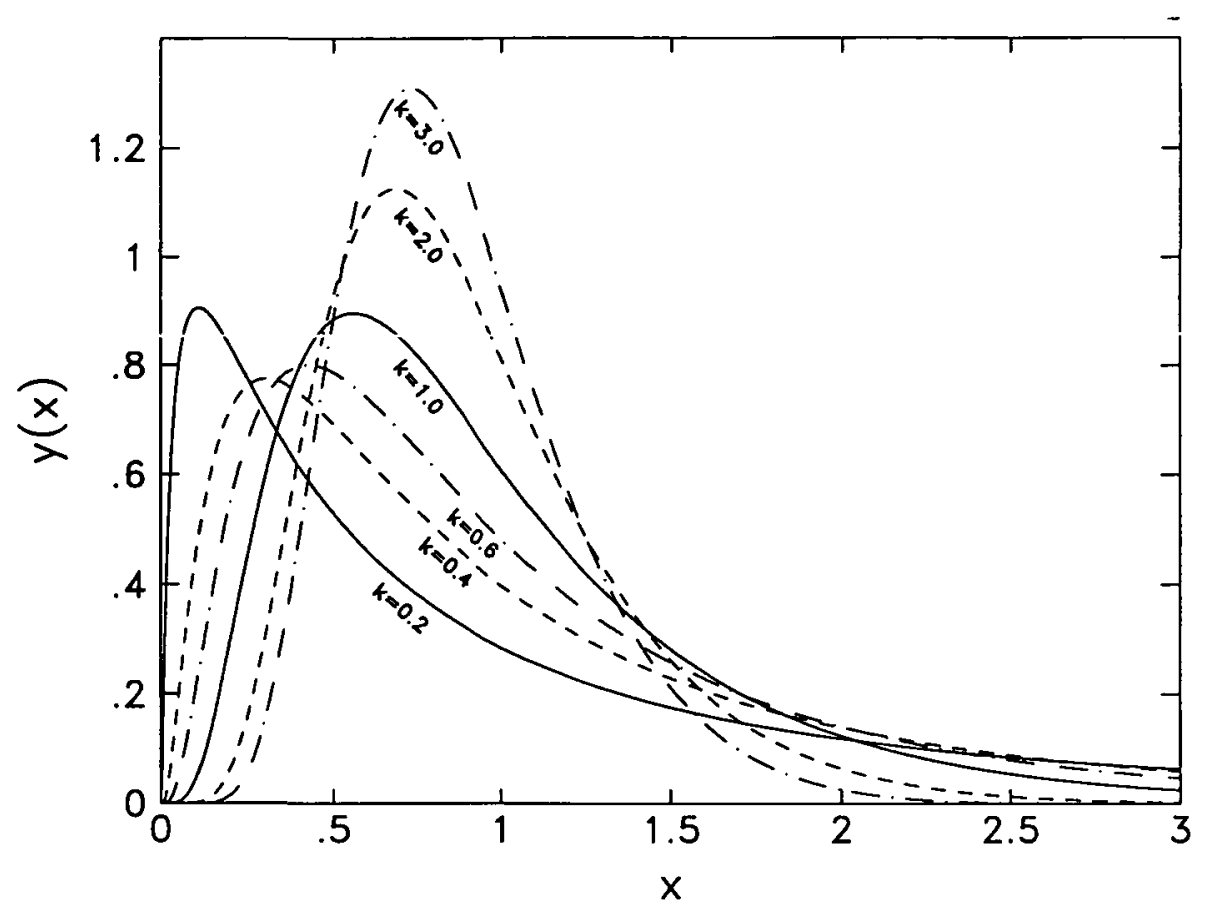

FIGURE 1. SSD probability density functions $y(x)$ for $g(x)=g x$ and $b(x)=b x^{k}$ for a range of values of $k$. The variables $a$ and $a$ have been fixed at 1 and 2 respectively.

delta function $\delta(x)$, and as $k \rightarrow \infty$ it approaches (Koch and Schaechter [6] the function

$$
y(x)= \begin{cases}a /(\alpha-1) x^{2}, & a / \alpha<x<a \\ 0, & \text { otherwise }\end{cases}
$$

3 A minimum size for Division: The case $b(x)=b x^{k} H\left(x-x_{1}\right)$

This case is the same as that discussed in the previous section, except that there is a minimum size $x_{1}>0$ below which cells do not divide. Substituting $b(x)=b x^{k} H\left(x-x_{1}\right), k>0,\left(b, x_{1}\right.$ constant) into equation (15) we get

$$
Z^{\prime}(x)=x^{k-1}\left(-a H\left(x-x_{1}\right) Z(x)+a \alpha^{k} H\left(\alpha x-x_{1}\right) Z(\alpha x)\right) .
$$

In this case we choose to couple (60) with the integral condition (17) as this simplifies to a condition dependent only on $Z(x)$ for $x \geq x_{1}$, namely

$$
\int_{x_{1}}^{\infty} x^{k-2} Z(x) d x=\frac{1}{a(\alpha-1)}
$$


It is convenient to solve the equation in three regions starting with the region $x>x_{1}$. We let

$$
Z(x)= \begin{cases}Z_{1}(x), x \geq x_{1} & \text { (region 1) } \\ Z_{2}(x), x_{1} / \alpha \leq x \leq x_{1} & \text { (region 2) } \\ Z_{3}(x), x \leq x_{1} / \alpha & \text { (region 3) }\end{cases}
$$

where continuity considerations require $Z_{1}\left(x_{1}\right)=Z_{2}\left(x_{1}\right)$ and $Z_{3}\left(x_{1} / \alpha\right)=$ $Z_{2}\left(x_{1} / \alpha\right)$.

\subsection{Region 1, $x \geq x_{1}$}

In this region, (60) becomes (21), so the solution is

$$
Z_{1}(x)=C \sum_{n=0}^{\infty} \frac{(-1)^{n} \alpha^{k n}}{\left(\alpha^{k}-1\right) \cdots\left(\alpha^{k n}-1\right)} e^{-a\left(\alpha^{n} x\right)^{k} / k}
$$

To find the constant $C$ we substitute $Z(x)=Z_{1}(x)$ into (61) above, giving

$$
C \int_{x_{1}}^{\infty}\left[\sum_{n=0}^{\infty} \frac{(-1)^{n} \alpha^{k n}}{\left(\alpha^{k}-1\right) \cdots\left(\alpha^{k n}-1\right)} x^{k-2} e^{-a \alpha^{n k} / x^{k} / k}\right] d x=\frac{1}{a(\alpha-1)}
$$

This time there is no difficulty in immediately interchanging the integral and summation operations as the integration starts at $x_{1}>0$ and therefore avoids the singularity at $x=0$ when $k \leq 1$. Making the change of variable $z=a \frac{\alpha^{n k} x^{k}}{k}$ in each integral leads to

$$
\frac{C}{a} \sum_{n=0}^{\infty} \frac{(-1)^{n} \alpha^{n}}{\left(\alpha^{k}-1\right) \cdots\left(\alpha^{k n}-1\right)}\left(\frac{a}{k}\right)^{1 / k} \int_{a a^{n k} x_{1}^{k} / k}^{\infty} z^{-1 / k} e^{-z} d z=\frac{1}{a(\alpha-1)}
$$

Hence we can write

$$
C=\left(\frac{k}{a}\right)^{1 / k} \frac{1}{(\alpha-1) S}
$$

where

$$
S=\sum_{n=0}^{\infty} \frac{(-1)^{n} \alpha^{n}}{\left(\alpha^{k}-1\right) \cdots\left(\alpha^{k n}-1\right)} \Gamma\left(1-\frac{1}{k}, \frac{a}{k} \alpha^{k n} x_{1}^{k}\right)
$$

and $\Gamma(c, x)=\int_{x}^{\infty} s^{c-1} e^{-s} d s$ is an incomplete gamma function.

In the particular case $k=1,(66)$ reduces to

$$
C=\frac{1}{a(\alpha-1) S}
$$

where

$$
S=\sum_{n=0}^{\infty} \frac{(-1)^{n} \alpha^{n}}{(\alpha-1) \cdots\left(\alpha^{n}-1\right)} E_{1}\left(a \alpha^{n} x_{1}\right)
$$

and $E_{1}$ is the exponential integral. 
3.2 Region, $x_{1} / \alpha \leq x \leq x_{1}$

In this region we have $H\left(x-x_{1}\right)=0$ and $H\left(\alpha x-x_{1}\right)=1$ so we may immediately integrate $(60)$ to obtain

$$
Z_{2}(x)=a \int_{x_{1}}^{\alpha x} x^{k-1} Z_{1}(s) d s .
$$

This integration can be carried out term hy term, again using the substitution $z=a \alpha^{n k} x^{k} / k$, to give

$$
Z_{2}(x)=C \sum_{n=0}^{\infty} \frac{(-1)^{n}}{\left(\alpha^{k}-1\right) \cdots\left(\alpha^{k n}-1\right)}\left[e^{-a \alpha^{n k} x_{1}^{k} / k}-e^{-a \alpha^{(n+1) k} x^{k} / k}\right],
$$

where the constant $C$ is the same as that in the expression for $Z_{1}(x)$ in (63).

3.3 Region 3, $x \leq x_{1} / a$

In this region (60) becomes

$$
Z_{3}^{\prime}(x)=0
$$

so as we have $Z_{3}\left(x_{1} / \alpha\right)=Z_{2}\left(x_{1} / \alpha\right)=0$ from (71) the solution is simply

$$
Z_{3}(x)=0
$$

as expected.

\subsection{Discussion and examples}

Combining the results above and noting $Z(x)=x^{2} y(x)$ the SSD in the case $g(x)=g x$ and $b(x)=b x^{k} H\left(x-x_{1}\right)$ is given by

$$
y(x)=\left\{\begin{array}{lr}
C / x^{2} \sum_{n=0}^{\infty} \frac{(-1)^{n} \alpha^{k n}}{\left(\alpha^{k}-1\right) \cdots\left(\alpha^{k n}-1\right)} e^{-a\left(\alpha^{n} x\right)^{k} / k}, \quad x \geq x_{1} \\
C / x^{2} \sum_{n=0}^{\infty} \frac{(-1)^{n}}{\left(\alpha^{k}-1\right) \cdots\left(\alpha^{k n}-1\right)}\left[e^{-a \alpha^{n k} x_{1}^{k} / k}-e^{-a \alpha(n+1) k_{x} k / k}\right], \\
0, \quad x \leq x_{1} / \alpha & x_{1} / \alpha \leq x \leq x_{1}
\end{array}\right.
$$

where

$$
C=(k a)^{1 / k} \frac{1}{(\alpha-1) S}
$$

and

$$
S=\sum_{n=0}^{\infty} \frac{(-1)^{n} \alpha^{n}}{\left(\alpha^{k}-1\right) \cdots\left(\alpha^{k n}-1\right)} \Gamma\left(1-\frac{1}{k}, \frac{a}{k} \alpha^{k n} x_{1}^{k}\right) .
$$

Figure 2 shows this piecewise function for $y(x)$ for a range of minimum sizes for cell division $x_{1}$, with $k=2, a=b / g=1$, and $\alpha=2$. The discontinuity in the slope of $y(x)$ at $x=x_{1}$ is a natural consequence of the step change in 


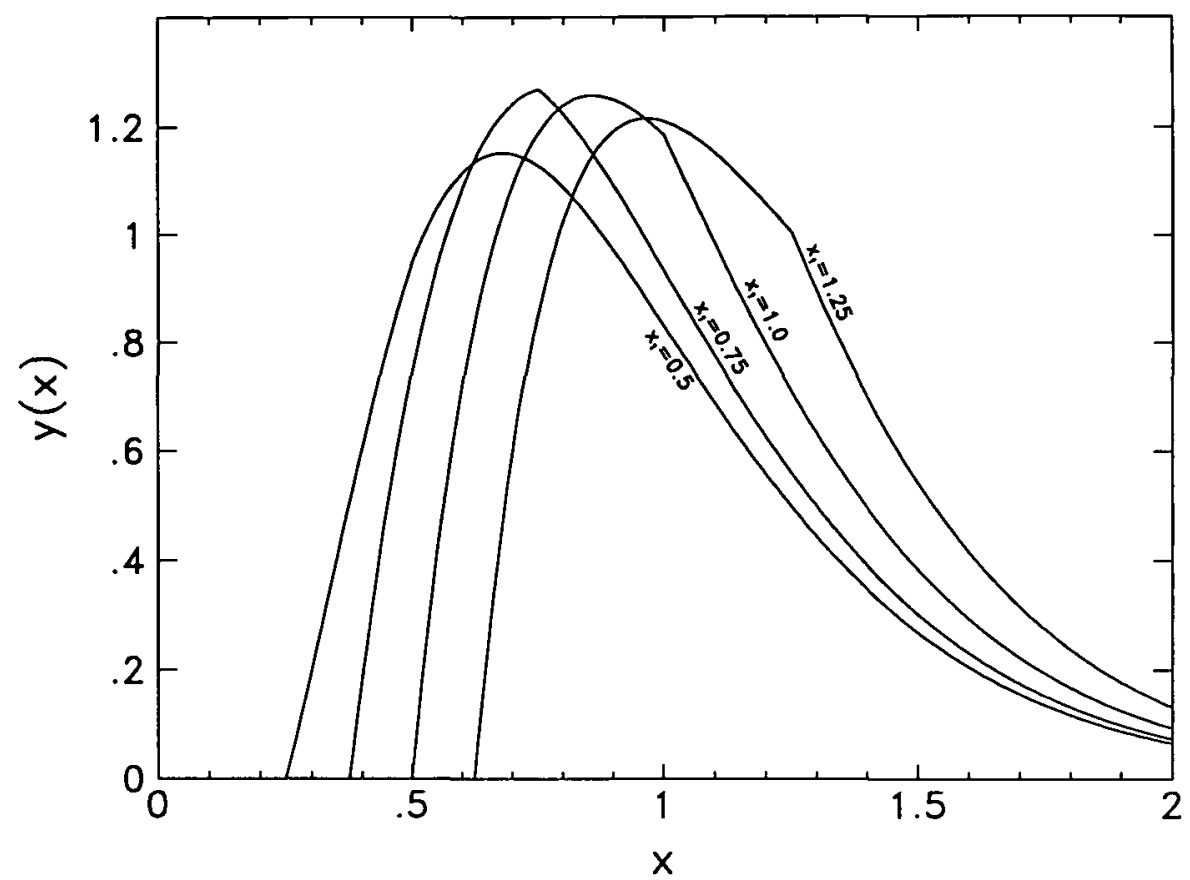

FIGURE 2. SSD probability density functions $y(x)$ for $g(x)=g x$ and $b(x)=b x^{k} H\left(x_{2}-x\right)$, with $k=2, a=1, \alpha=2$, and a range of values of $x_{1}$.

the probability of cell division $b(x)$ (from 0 to $b x_{1}^{k}$ ) at this size. The form of $y(x)$ for small values of $x_{1}$ approaches the form given in figure 1 for $k=2$.

\section{A minimum and maximum size for cell division}

Consider the case where $g(x)=g x$ and there exists a minimum size for cell division $x_{1}$ as in the previous section, and there is also a maximum cell size $x_{2}\left(0<x_{1}<x_{2}\right)$ such that all cells divide before they reach size $x_{2}$. This means we are considering non-negative forms of $a(x)=b(x) / g x$ such that

$$
\begin{aligned}
a(x)=0, & x \leq x_{1}, \\
a(x) \rightarrow \infty, & x \rightarrow x_{2}^{-},
\end{aligned}
$$

and we will assume $y(x) \equiv 0$ for $x>x_{2}$. For reasons that will become apparent later we will in fact restrict our attention to forms of $a(x)$ which are integrable over all closed regions $[c, d]$ such that $c, d<x_{2}$, but with a 
non-integrable singularity at $x_{2}$, so

$$
\int_{c}^{x_{2}} a(x) d x=\infty
$$

for any $c<x_{2}$.

\subsection{The solution method}

The solution, $Z(x)$, to (15) can be constructed as follows. Firstly, let $m$ be the largest integer such that $x_{1}<x_{2} \alpha^{-m}$. In typical cell populations, $m$ is usually quite small-less than 3 . Tyson and Diekman [8] give the solution for the special case $m=0$ and $\alpha=2$, and show how in this case solutions may be obtained for forms of $g(x)$ other than $g(x)=g x$ by treating $Q$ (see section 1) as an unknown constant to be found numerically. The method outlined here could be similarly extended to more general forms of $g(x)$.

We split $Z(x)$ into regions so that

$$
Z(x)= \begin{cases}Z_{0}(x), & x \geq x_{2} \\ Z_{k}(x), & x_{2} \alpha^{-k} \leq x<x_{2} \alpha^{-k+1}, 1<k \leq m+1 .\end{cases}
$$

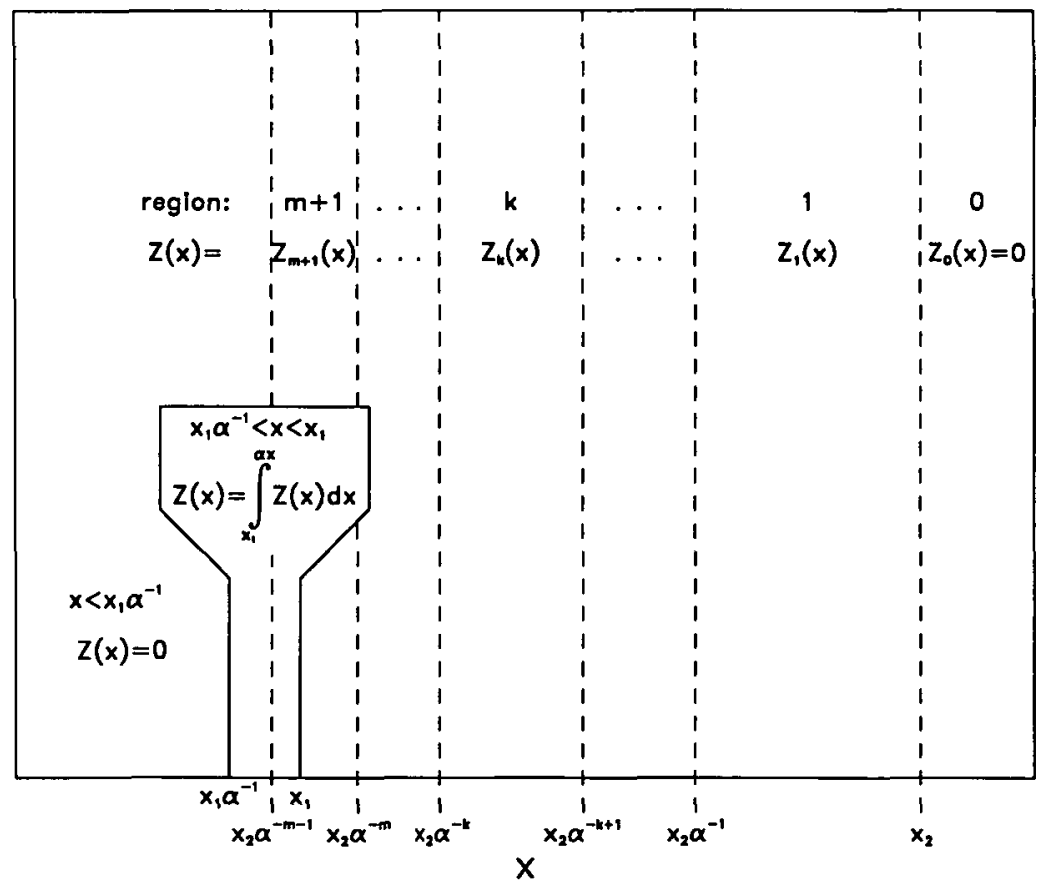

FIGURE 3. Regions used in solving for the SSD. $Z_{1}(x)$ is found first using $(81), Z_{2}(x)$ to $Z_{m+1}(x)$ are found iteratively using (82), then finally $Z(x)$ for $x<x_{1}$ is found using (83). 
This scheme is shown in Fig. 3. Note that as no cell division occurs below size $x_{1}$, no new cells can appear of size less than $x_{1} \alpha^{-1}$, so we must have $z(x)=0$ for $x<x_{1} \alpha^{-1}$. We now write (15) in the form

$$
Z_{k+1}^{\prime}(x)=-a(x) Z_{k+1}(x)+\alpha a(\alpha x) Z_{k}(\alpha x), \quad k \geq 0 .
$$

This equation can then be used iteratively to find $Z(x)$ over the whole range of sizes of interest. We know $Z_{0} \equiv 0$ (because $y(x)=0$ for $x>x_{2}$ ), so it follows from (80) that

$$
Z_{1}(x)=C e^{-A(x)}
$$

where $C$ is a constant to be evaluated, and $A^{\prime}(x)=a(x)$. If we now assume that the solution in region $k$ is known, then (80) becomes a simple first-order ODE in $Z_{k+1}(x)$, and the solution is

$$
\begin{aligned}
Z_{k+1}(x)= & \exp \left(\int_{x}^{x_{2} \alpha^{-k}} a(s) d s\right) Z_{k}\left(x_{2} \alpha^{-k}\right) \\
& -\int_{\alpha x}^{x_{2} \alpha^{1-k}} \exp \left(\int_{x}^{s a^{-1}} a\left(s^{\prime}\right) d s^{\prime}\right) a(s) Z_{k}(s) d s,
\end{aligned}
$$

where the limits of the integration have been fixed by the continuity requirement $Z_{k+1}\left(x_{2} \alpha^{-k}\right)=Z_{k}\left(x_{2} \alpha^{-k}\right)$. Hence we can deduce the solution $Z_{k}(x)$ for all $k \geq 1$, then calculate the constant $C$ from the either of the integral conditions (16) or (17). The desired SSD can then be simply calculated from $y(x)=x^{-2} Z(x)$.

Two points are worth noting in this solution process. Firstly, from (81), continuity of $Z_{1}$ with $Z_{0}$ at $x=x_{2}$ for non-zero $C$ requires that $A\left(x_{2}\right)=\infty$, so $x_{2}$ must be a non-integrable singularity of $a(x)$ as pointed out earlier. The alternative would be to allow a discontinuity in $Z(x)$ at $x_{2}$, in which case $y(x)$ would be discontinuous at $x=x_{2} \alpha^{-k}$ for $0 \leq k \leq m+1$, so the argument involving continuity used above would be invalid. Equation (81) must clearly be used in place of (82) for $k=0$ because the first term in (82) becomes a product of infinity and zero. Secondly, once the solution has been found in the size region between $x_{1}$ and $x_{2}$ using the above process, the solution in the region $x<x_{1}$ is most simply found by noting that $a(x) \equiv 0$, so the integral form of the equation (18) can be used in the form

$$
Z(x)=\int_{x_{1}}^{\alpha x} a(s) Z(s) d s, \quad x<x_{1} .
$$

It is clear from this formulation that we will have $Z(x)=0$ for $x<x_{1}$ as expected, with continuity of $Z(x)$ at $x=x_{1}$. 


\subsection{An example}

As an analytical example of this process, consider the simplest form of $a(x)$ which satisfies the conditions (77), namely

$$
a(x)= \begin{cases}1 /\left(x_{2}-x\right), & x_{1}<x<x_{2} \\ 0, & x<x_{1},\end{cases}
$$

which corresponds to a birth rate $b(x)$ of

$$
b(x)=\left\{\begin{array}{l}
g x /\left(x_{2}-x\right), \quad x_{1}<x<x_{2} \\
0, x<x_{1}
\end{array}\right.
$$

where $g$ is the constant in $g(x)=g x$. We will choose $x_{1}=\alpha^{-2} x_{2}$ exactly, as this is the simplest choice which involves use of all of the equations (81), (82), and (83). In region 1 we then find, using (81),

$$
\begin{aligned}
Z_{1}(x) & =C e^{-\int 1 /\left(x_{2}-x\right) d x} \\
& =C x_{2}\left(1-x / x_{2}\right) .
\end{aligned}
$$

In region 2, using (82) we obtain

$$
Z_{2}(x)=C x_{2}\left(1-x / x_{2}\right)\left[1-\alpha \log \left(1-x / x_{2}\right)+\alpha \log \left(1-\alpha^{-1}\right)\right] .
$$

Applying integral condition (17) we get

$$
\int_{x_{2} \alpha^{-2}}^{x_{2} \alpha^{-1}} \frac{Z_{2}(x)}{x\left(x_{2}-x\right)} d x+\int_{x_{2} \alpha^{-1}}^{x_{2}} \frac{Z_{1}(x)}{x\left(x_{2}-x\right)} d x=\frac{1}{\alpha-1}
$$

which leads to

$$
C=\left[(\alpha-1)\left[\left(2+\alpha \log \left(1-\alpha^{-1}\right)\right) \log \alpha+\alpha\left(S\left(\alpha^{-1}\right)-S\left(\alpha^{-2}\right)\right)\right]\right]^{-1},
$$

where $S(u)=\sum_{n=1}^{\infty} u^{n} / n^{2}$.

We now use (82) to find the solution in the region $x<x_{1}$ as

$$
\begin{aligned}
Z_{3}(x)=C x_{2} \alpha[ & \left(1+\alpha \log \left(1-\alpha^{-1}\right)\right)\left[\frac{x}{x_{2}}-\alpha^{-3}\right] \\
+\left(1-\alpha \frac{x}{x_{2}}\right) & {\left[\log \left(1-\alpha \frac{x}{x_{2}}\right)-1\right] } \\
& \left.-\left(1-\alpha^{-2}\right)\left[\log \left(1-\alpha^{-2}\right)-1\right]\right]
\end{aligned}
$$




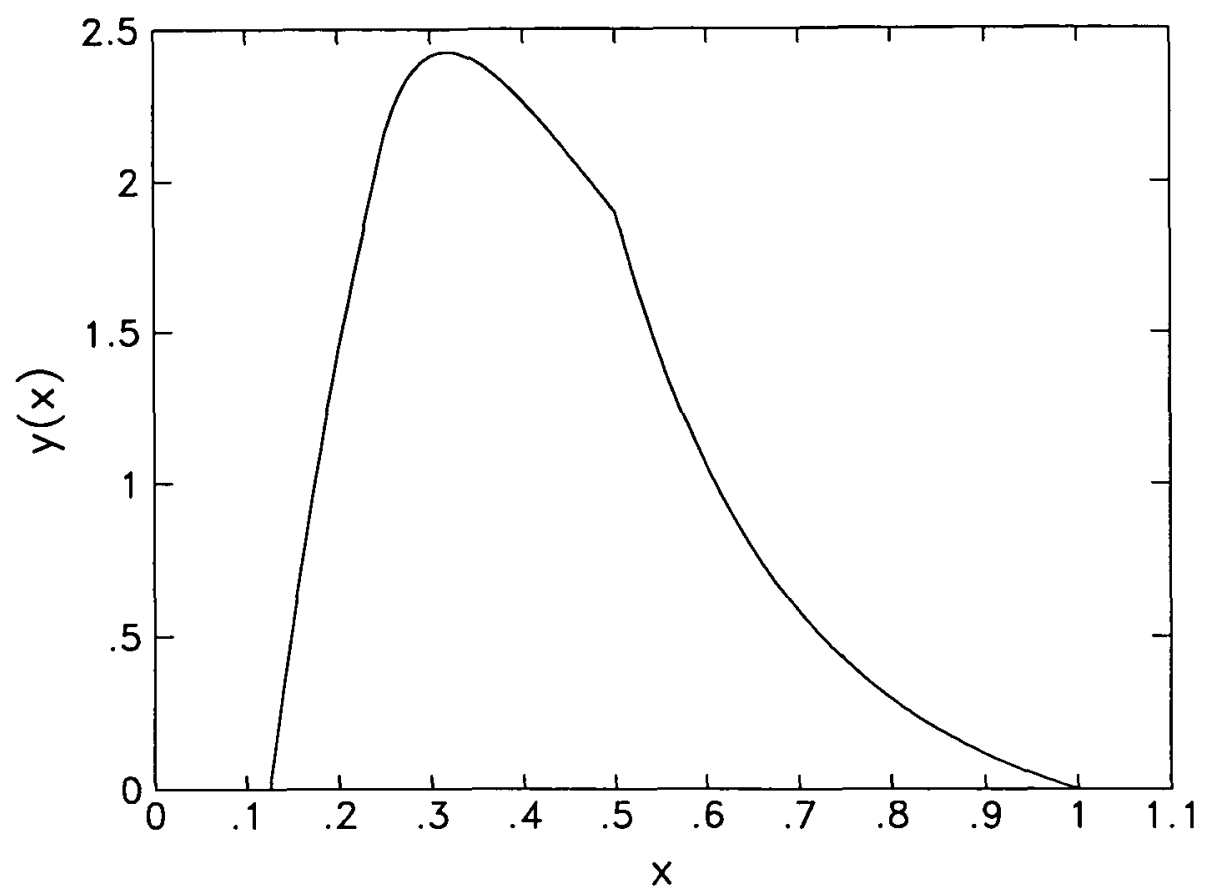

FIGURE 4. The SSD probability density functions $y(x)$ for $a(x)=b(x) / g x=(1-x)^{-1}$. $H(x-0.25)$ and $\alpha=2$.

Now using $u(x)=x^{-2} Z(x)$ we can write the SSD $y(x)$ as

$$
y(x)=\left\{\begin{array}{l}
0, \quad x \leq x_{2} \alpha^{-3} \\
\frac{C x_{2}}{x^{2}} \cdot \alpha\left[\left(1+\alpha \log \left(1-\alpha^{-1}\right)\right)\left[\frac{x}{x_{2}}-\alpha^{-3}\right]\right. \\
+\left(1-\alpha \frac{x}{x_{2}}\right)\left[\log \left(1-\alpha \frac{x}{x_{2}}\right)-1\right], \quad x_{2} \alpha^{-3}<x \leq x_{2} \alpha^{-2} \\
-\left(1-\alpha^{-2}\left[\log \left(1-\alpha^{-2}\right)-1\right]\right], \\
\frac{C x_{2}}{x^{2}}\left(1-\frac{x}{x_{2}}\right)\left[1-\alpha \log \left(1-\frac{x}{x_{2}}\right)+\alpha \log \left(1-\alpha^{-1}\right)\right], \\
\frac{C x_{2}}{x^{2}}\left(1-\frac{x}{x_{2}}\right), \quad x_{2} \alpha^{-1}<x \leq x_{2} \alpha^{-1} \\
0, \quad x>x_{2} .
\end{array}\right.
$$

where $C$ is given by (89).

Fig. 4 shows this function with binary fission $(\alpha=2)$ and with $x_{2}$ equal to one size unit. Substitution is straightforward apart from the calculation of $S(0.5)$ and $S(0.25)$ required to find $C$. Jolley [4] gives

$$
S(0.5)=\frac{\pi^{2}}{12}-\frac{(\log 2)^{2}}{2}
$$


but the only way to find $S(0.25)$ seems to be to substitute into the series, which actually converges very rapidly in this case. From the form of (91) it is clear that $x_{2}$ is just a scaling factor, so the solution for other values of $x_{2}$ is simply obtained by relabelling the horizontal and vertical axes in Fig. (4) as $x / x_{2}$ and $x_{2} y(x)$ respectively.

\section{Acknowledgement}

Thanks are due to P. W. Gandar (Plant Physiology Division, DSIR, Palmerston North, New Zealand) for providing the motivation for this work and for a number of constructive comments during the preparation of this paper.

\section{References}

[1] G. E. Andrews, "Number Theory", (W. B. Saunders, Phil. 1971).

[2] J. F. Collins and M. H. Richmond, "Rate of Growth of Bacillus Cereus Between Divisions", J. gen. Microbiol. 28 (1962), 15-33.

[3] A. J. Hall and G. C. Wake, "A Functional Differential Equation Arising in the Modelling of Cell Growth", J. Austral. Math. Soc. Ser. B 30 (1989) 424-435.

[4] L. B. W. Jolley, "Summation of Series", (Dover, N.Y. 1961).

[5] T. Kato and J. B. Mcleod, "The Functional Differential Equation $y^{\prime}(x)=a y(\lambda x)+b y(x)$ ", Bull. Amer. Math. Soc. 77 (1971) 891-937.

[6] A. L. Koch and M. Schaechter, "A Model for Statistics of the Cell Division Process", J. gen. Microbiol. 29 (1962) 435-454.

[7] E. O. Powell, "A Note on Koch \& Schaechter's Hypothesis about Growth and Fission of Bacteria", J. gen. Microbiol. 37 (1964) 231-249.

[8] J. Tyson and O. Diekmann, "Sloppy Size Control of the Division Cycle", J. theor. Biol. 118 (1986) 405-426.

\section{Appendix: Identities involving $K\left(\alpha^{k}, \beta\right)$}

If we take the well-known identity (for example, see [1])

$$
\sum_{n=0}^{\infty} \frac{q^{n(n-1) / 2} z^{n}}{(1-q)\left(1-q^{2}\right) \cdots\left(1-q^{n}\right)}=\prod_{n=0}^{\infty}\left(1+z q^{n}\right),
$$

and substitute $q=\alpha^{-k}$ we get

$$
\sum_{n=0}^{\infty} \frac{\alpha^{k n} z^{n}}{\left(\alpha^{k}-1\right)\left(\alpha^{2 k}-1\right) \cdots\left(\alpha^{n k}-1\right)}=\prod_{n=0}^{\infty}\left(1+z \alpha^{-k n}\right) .
$$


Substituting $\beta=-\alpha^{k} z$ gives

$$
\sum_{n=0}^{\infty} \frac{(-1)^{n} \beta^{n}}{\left(\alpha^{k}-1\right) \cdots\left(\alpha^{k n}-1\right)}=\prod_{n=1}^{\infty}\left(1-\beta \alpha^{-k n}\right)
$$

so that using the definition of $K$ given in (36) we obtain

$$
K\left(\alpha^{k}, \beta\right)=\prod_{n=1}^{\infty}\left(1-\beta \alpha^{-k n}\right)
$$

Substituting $\beta=\alpha^{m}$ into (96) then gives

$$
K\left(\alpha^{k}, \alpha^{m}\right)=\prod_{n=1}^{\infty}\left(1-\alpha^{-k(n-1)+m-k}\right),
$$

so

$$
K\left(\alpha^{k}, \alpha^{m}\right)=\left(1-\alpha^{m-k}\right) K\left(\alpha^{k}, \alpha^{m-k}\right)
$$

